\title{
The positivity of G-protein-coupled receptor-30 (GPR 30), an alternative estrogen receptor is not different between type 1 and type 2 endometrial cancer
}

 \\ ${ }^{1}$ Department of Pathology, Wuxi No2 People's Hospital, Nanjing Medical University, Wuxi, China \\ ${ }^{2}$ Department of Pathology, Wuxi Maternity and Children Hospital, Nanjing Medical University, Wuxi, China \\ ${ }^{3}$ The Hospital of Obstetrics and Gynaecology, Fudan University, Shanghai, China \\ ${ }^{4}$ Centre for Reproductive Medicine, Shanghai First Maternity and Infant Hospital, Tongji University School of Medicine, \\ Shanghai, China \\ ${ }^{5}$ Department of Obstetrics and Gynaecology, The University of Auckland, Auckland, New Zealand \\ *These authors contributed equally to this work
}

Correspondence to: Qi Chen, email: q.chen@auckland.ac.nz

Keywords: endometrial cancer, GPR30, estrogen receptor, menopause, type 1 and type 2

Received: February 13, 2017 Accepted: June 04, 2017 Published: June 17, 2017

Copyright: Wan et al. This is an open-access article distributed under the terms of the Creative Commons Attribution License 3.0 (CC BY 3.0), which permits unrestricted use, distribution, and reproduction in any medium, provided the original author and source are credited.

\section{ABSTRACT}

It is well-known that the clinical outcomes are different between type 1 (estrogen dependent) and type 2 (estrogen independent) endometrial cancer. Studies have suggested that the estrogen receptor (ER) is positively correlated with endometrial cancer survival, however we previously reported that there is no difference in the positivity of ER as well as sex hormone levels between subtypes of cancer. G-proteincoupled receptor-30 (GPR 30), an alternative estrogen receptor has been suggested to be negatively correlated with clinical outcomes of endometrial cancer. In this study we investigated whether the positivity of GPR30 is different between subtypes of cancer. The immunostaining of GPR30 and ER was examined and analysed in 128 cases taking into account menopausal status. Overall, $105(82 \%)$ cases were GPR30 positive and $118(92 \%)$ cases were ER positive. The positivity of GPR30 in type 1 endometrial cancer $(83 \%)$ was not statistically different to type 2 endometrial cancer $(78 \%)$. In addition, intensity of immunostaining of GPR30 in type 1 endometrial cancer was also not different to type 2 endometrial cancer quantified by semi-quantitative analysis $(p=0.268)$. Menopausal status was not associated with the positivity of GPR30 in both type 1 and type 2 endometrial cancer. Furthermore, the positivity and intensity of immunostaining of GPR30 were not correlated with the positivity and intensity of immunostaining of ER in endometrial cancer $(p=0.689)$. Our data further confirm that type 2 endometrial cancer may not be completely estrogen independent, and suggest that type 1 and type 2 endometrial cancer may have similar pathogenesis.

\section{INTRODUCTION}

Endometrial cancer has recently been a major gynaecological cancer in developed countries and causes more than 10,000 deaths in the United States yearly (American Cancer Society: Cancer Facts and Figures 2016). The exact causes of endometrial cancer are still unclear, however unopposed endometrial estrogen exposure, such as estrogen replacement therapy during menopause has been suggested to be associated with increased risk of developing this disease [1]. Endometrial cancer is traditionally divided into estrogen dependent (type 1) and estrogen independent (type 2) [2], although a new classification of endometrial cancer has recently 
been reported that includes four pathological subtypes of endometrial cancer based on molecular signatures [3]. Type 1 endometrial cancer is thought to be caused by excess estrogen, while type 2 endometrial cancer was not.

Estrogen and progesterone exert their effect through intra-and extra-nuclear receptors. It is well documented that the positivity of estrogen receptor (ER) and progesterone receptor (PR) is positively associated with the prognosis of endometrial cancer, including the survival rate and survival time $[4,5]$. Although type 1 endometrial cancer has a better survival rate with treatment, while type 2 has a poorer prognosis with an aggressive form of the disease [2], our recent studies have found that there is no difference in the positivity of ER or PR as well as the sex hormone levels between type 1 and type 2 endometrial cancer [6, 7]. In addition, a study has hypothesised that type 2 endometrial cancer may not be completely estrogen-independent because both type 1 and type 2 endometrial cancer share many common risk factors [8]. This suggests that another factor(s) may be involved in causing the difference in clinical outcomes between type 1 and type 2 endometrial cancer.

G-protein-coupled receptor-30 (GPR 30), an alternative intra-cellular estrogen receptor was identified in 2005 and is able to mediate estrogen action [9, 10]. Unlike subunits of ER (ER $\alpha$ and $E R \beta)$ that function as estrogen- activated transcription factors in the nucleus and do not influence gene transcription [11], GPR30 is a transmembrane estrogen receptor which is involved in the rapid nongenomic effect of estrogen [reviewed in [12-14]] and is a specific receptor for $17 \beta$-estradiol which is a most potent estrogen subtype [12]. GPR30 is widely overexpressed in a number of cancer cells including endometrial cancer cells [15-17] and has been suggested to be a novel indicator of clinical outcomes of endometrial cancer [18]. This potentially suggests that the positivity of GPR30 may be different between type 1 and type 2 endometrial cancer.

Therefore, this study aimed to investigate the positivity of GPR30 in endometrium between type 1 and type 2 endometrial cancer taking into account menopausal status and whether the positivity of GPR30 is correlated with the positivity of ER.

\section{RESULTS}

\section{Clinical characteristics of the study population}

The clinical and histological characteristics of study participants are summarised in Table 1. The median age of patients at diagnosis was 56 (range 29-82) years old. Of 128 patients, $100(78 \%)$ were diagnosed with type 1 endometrial cancer, and 44 (34\%) patients were diagnosed before menopause. There was no statistical difference in the median age between premenopausal women with type 1 (55 range from 29 to 82 years) and type 2 endometrial cancer (57 range from 35 to 71 years) at diagnosis. There was also no statistical difference in the median age between postmenopausal women with type 1 (61 range from 46 to 82 years) and type 2 endometrial cancer (63 range from 47 to 71 years) at diagnosis.

\section{GPR30 was expressed in the luminal or basal surface of epithelium}

Overall, $105(82 \%)$ cases were GPR30 positive. We then investigate the localization of GPR30. The immunostaining of GPR30 was performed by immunohistochemistry (Figure 1). GPR30 mainly expressed in the luminal (Figure 1A and 1B) or basal surface of epithelium (Figure 1C and 1D) in either type 1 or type 2 endometrial cancer. Semi-quantitative analysis of the overall immunohistochemistry staining showed that there was no difference in the intensity of immunostaining of GPR 30 between type 1 and type 2 endometrial cancer (Figure 1E, $p=0.268$ ).

\section{The positivity of GPR30 is not different between type 1 and type 2 endometrial cancer}

We then analysed the positivity of GPR30 between two subtypes of endometrial cancer. In type 1 endometrial cancer, $83(83 \%)$ cases were GPR 30 positive, whereas in type 2 endometrial cancer, 19 (78\%) cases were GPR30 positive respectively (Table 2). There was no statistical difference in the positivity of GPR30 between type 1 and type 2 endometrial cancer $(p=0.391)$.

Menopausal status is one of the risk factors for developing endometrial cancer, we then compared the positivity of GPR30 in patients before menopause or after menopause according to the cancer types (Table $3)$. In premenopausal women, $32(88.8 \%)$ cases with type 1 endometrial cancer were GPR30 positive, and $7(87.5 \%)$ cases with type 2 endometrial cancer were GPR30 positive. There was no statistical difference in the positivity of GPR30 between type 1 and type 2 endometrial cancer in premenopausal women $(p=0.999)$. Similarly, in postmenopausal women, 51 (80\%) cases with type 1 endometrial cancer were GPR30 positive and $15(75 \%)$ cases with type 2 endometrial cancer were GPR30 positive. There was also no statistical difference in the positivity of GPR30 between type 1 and type 2 endometrial cancer in premenopausal women $(p=0.756)$.

\section{The positivity of GPR30 in endometrial cancer is not associated with menopausal status}

We further investigated whether the positivity of GPR30 is associated with menopausal status taking into account cancer subtypes. As shown in Table 4, in type 1 endometrial cancer $(n=100), 32(88.8 \%)$ cases were GPR30 positive in premenopausal women. $51(80 \%)$ 
cases were GPR30 positive in postmenopausal women. There was no difference in the positivity of GPR30 in type 1 endometrial cancer between premenopausal and postmenopausal patients (Table $4, p=0.283$ ). In type 2 endometrial cancer $(n=28), 7(87.5 \%)$ cases were
GPR30 positive in premenopausal women. 15 (75\%) cases were GPR30 positive in postmenopausal women. There was also no difference in the positivity of GPR30 in type 2 endometrial cancer between premenopausal and postmenopausal patients (Table $4, p=0.634$ ).
Type 1 endometrial cancer


Type 2 endometrial cancer
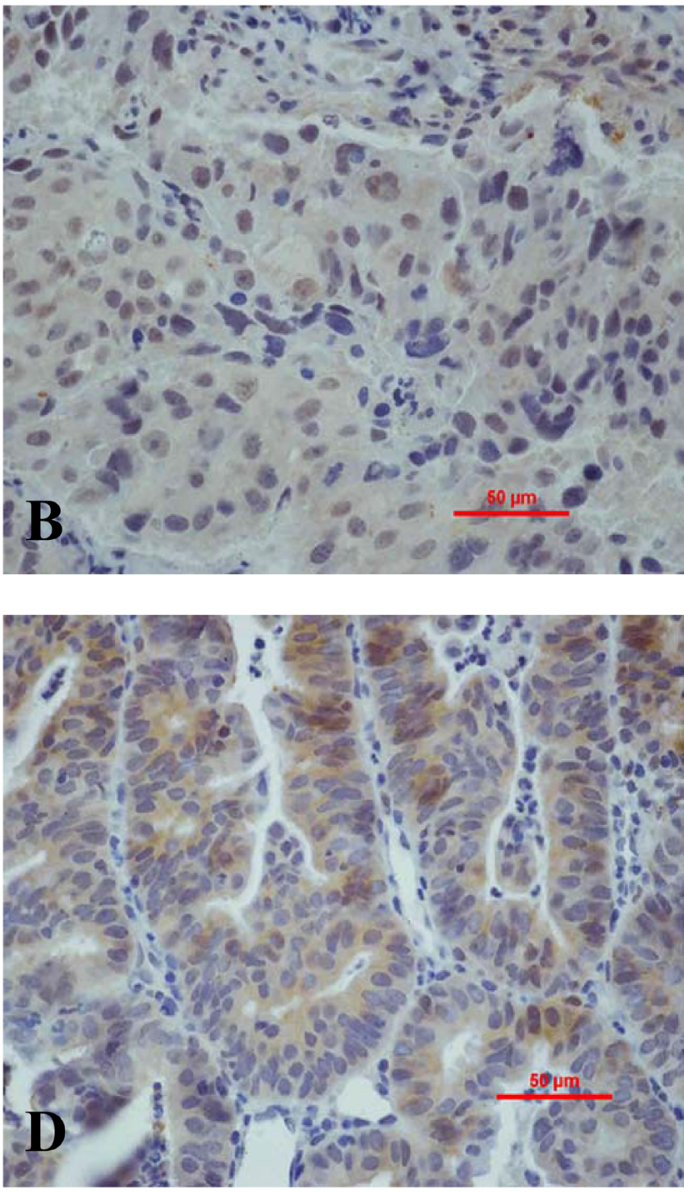

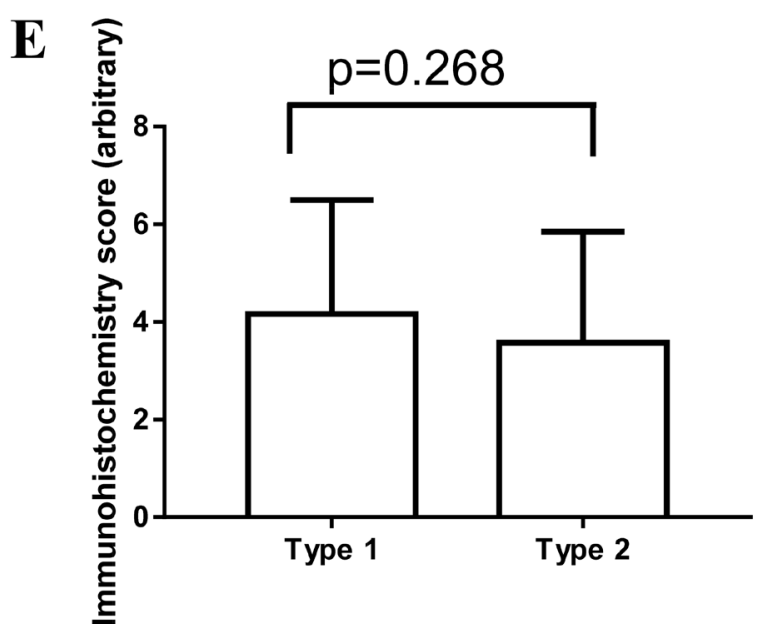

Figure 1: Representative immunohistochemistry images showing the immunostaining of GPR30 in type 1 (left) and type 2 (right) endometrial cancer in the luminal (A, B) or endometrial glands $(\mathbf{C}, \mathbf{D})$. Semi-quantitation of the overall immunohistochemical analysis indicated there was no difference in intensity of staining between type 1 and type 2 endometrial cancer $(\mathbf{E}, p=0.268)$. 
Table 1: Clinical characteristics of the study population

\begin{tabular}{|l|c|}
\hline \multicolumn{2}{|c|}{ Women with endometrial cancer $(N=128)$} \\
\hline Premenopause (number, \%) & $56(29-82)$ \\
\hline Post- menopause (number, \%) & $44(34 \%)$ \\
\hline Type 1(number, \%) & $84(66 \%)$ \\
\hline Type 2 (number, \%) & $100(78 \%)$ \\
\hline
\end{tabular}

Table 2: The expression of estrogen receptor (ER) or progesterone receptor (PR) in endometrial cancer

\begin{tabular}{|l|c|c|c|}
\hline \multicolumn{2}{|c}{ Type 1 $(\boldsymbol{n}=\mathbf{1 0 0})$} & Type 2 $(\boldsymbol{n}=\mathbf{2 8})$ & $\boldsymbol{P}$ value \\
\hline GPR30 positive (number, \%, lower, upper & $83(83 \%)$ & $22(78 \%)$ & 0.391 \\
CL) & $(75 \%, 90 \%)$ & $(59 \%, 91 \%)$ & \\
\hline
\end{tabular}

Table 3: The expression of GPR30 in endometrial cancer between cancer types according to menopausal status

\begin{tabular}{|l|c|c|c|}
\hline \multicolumn{1}{c|}{ premenopause } & Type 1 $(\boldsymbol{n}=\mathbf{3 6})$ & Type 2 $(\boldsymbol{n}=\mathbf{8})$ & $\boldsymbol{P}$ value \\
\hline GPR30 positive (number, \%) & $32(88.8 \%)$ & $7(87.5 \%)$ & $p=0.999$ \\
\hline postmenopause & Type 1 $(n=64)$ & Type 2 $(n=20)$ & \\
\hline GPR30 positive (number, \%) & $51(80 \%)$ & $15(75 \%)$ & $P=0.756$ \\
\hline
\end{tabular}

Table 4: The expression of GPR30 in endometrial cancer between premenopause and postmenopause according to subtypes of endometrial cancer

\begin{tabular}{|l|c|c|c|}
\hline \multicolumn{1}{c|}{ Type 1 $(\boldsymbol{n}=\mathbf{1 0 0})$} & Premenopause $(\boldsymbol{n}=\mathbf{3 6})$ & Postmenopause $(\boldsymbol{n}=\mathbf{6 4})$ & $\boldsymbol{P}$ value \\
\hline GPR30 positive (number, \%) & $32(88.8 \%)$ & $51(80 \%)$ & $p=0.281$ \\
\hline Type 2 $(n=28)$ & Premenopause $(n=8)$ & Postmenopause $(n=20)$ & \\
\hline GPR30 positive (number, \%) & $7(87.5 \%)$ & $15(75 \%)$ & $P=0.634$ \\
\hline
\end{tabular}

The positivity of GPR30 is not correlated with ER positivity in endometrial cancer

Studies have indicated that GPR30 is an alternative estrogen receptor $[9,10]$. We then investigated the correlation between the positivity of GPR30 and ER in endometrial cancer. The overall positivity of ER or GPR30 was $92 \%$ (118 of 128 cases) or $82 \%$ (105 of 128 cases), respectively. In GPR 30 negative cases $(n=23)$, there was $22(96 \%)$ cases that were ER positive. While, in GPR30 positive cases $(n=105)$, there were $96(91 \%)$ cases that were ER positive. There was no difference in the positivity of ER between GPR30 positive and GPR30 negative cases $(p=0.685$, Table 5).

In addition, in the cases of GPR30 with intensity of immunostaining $1+(n=28)$, the percentage of ER with intensity of staining $1+$, or $2+$ or $3+$ was $19 \%$ or $25 \%$ or $46 \%$ respectively, which was not different among the groups (Table 6). In the cases of GPR30 with intensity of immunostaining $2+(n=41)$, the percentage of ER with intensity of immunostaining $1+$, or $2+$ or $3+$ was $30 \%$ or $27 \%$ or $31 \%$ respectively, which was also not different among the groups (Table 6). In the cases of GPR30 with intensity of immunostaining $3+(n=36)$, the percentage of ER with intensity of immunostaining $1+$, or $2+$ or $3+$ was $25 \%$ or $39 \%$ or $33 \%$ respectively, which was also not different among the groups (Table 6).

\section{DISCUSSION}

GPR30, an alternative estrogen receptor has been reported to mediate the proliferative effects of estrogen in endometrial, ovarian and breast cancer cells [19]. The overexpression of GPR30 has been suggested to be negatively correlated with the clinical outcomes of endometrial cancer including survival rate and prognosis [18]. In our current study we found that the overall positivity 
Table 5: The correlation between the positivity of GPR30 and ER in endometrial cancer

ER positive (number, \%)

\begin{tabular}{|l|c|}
\hline GPR30 negative $(n=23)$ & $22(96 \%)$ \\
\hline GPR30 positive $(n=105)$ & $96(91 \%)$ \\
\hline$P$ value & 0.689 \\
\hline
\end{tabular}

\section{Table 6: The correlation between the expression (intensity of staining) of GPR30 and ER in} endometrial cancer

\begin{tabular}{|c|c|c|c|c|}
\hline & & \multicolumn{3}{|c|}{ ER expression (number, \%, lower, upper CL) } \\
\hline & & $1+$ & $2+$ & $3+$ \\
\hline \multirow[t]{3}{*}{ GPR30 expression } & $1+(n=28)$ & $\begin{array}{c}5(19 \%) \\
(6 \%, 36 \%)\end{array}$ & $\begin{array}{c}7(25 \%) \\
(10 \%, 44 \%)\end{array}$ & $\begin{array}{c}13(46 \%) \\
(27 \%, 66 \%)\end{array}$ \\
\hline & $2+(n=41)$ & $\begin{array}{c}12(30 \%) \\
(16 \%, 45 \%)\end{array}$ & $\begin{array}{c}11(27 \%) \\
(14 \%, 42 \%)\end{array}$ & $\begin{array}{c}13(31 \%) \\
(18 \%, 48 \%)\end{array}$ \\
\hline & $3+(n=36)$ & $\begin{array}{c}9(25 \%) \\
(12 \%, 42 \%)\end{array}$ & $\begin{array}{c}14(39 \%) \\
(23 \%, 56 \%)\end{array}$ & $\begin{array}{c}12(33 \%) \\
(18 \%, 50 \%)\end{array}$ \\
\hline
\end{tabular}

of GPR30 in endometrial cancer was $82 \%$ in Chinese population. Another study reported that the overall positivity of GPR30 in endometrial cancer was $87 \%$ in Caucasians which was higher than Chinese population [18]. Ethnicity is one of the risk factors for developing endometrial cancer and the study suggested that Asian women with endometrial cancer have improved clinical outcomes and better survival rate compared to non-Asian women [20]. This may be associated with lower positivity of GPR30 in Chinese (Asian) population with endometrial cancer.

Endometrial cancer traditional divides type 1 (estrogen dependent) and type 2 (estrogen independent) cancer. It is well-known that type 2 endometrial cancer has poorer clinical outcomes and prognosis compared to type 1 endometrial cancer, however to date whether there is a difference in the positivity of GPR 30 between type 1 and type 2 endometrial cancer has not been investigated yet. In this study we interestingly found that there was no statistical difference in the positivity of GPR30 between type 1 and type 2 endometrial cancer ( $83 \%$ vs $78 \%$ ). We further examined whether these is a difference in intensity of immunostaining of GPR30 between type 1 and type 2 endometrial cancer. However we found that there was also no difference in the intensity of immunostaining of GPR30 between type 1 and type 2 endometrial cancer. Taken together our data suggests that both positivity and intensity of immunostaining of GPR30 may not be related to the subtypes of endometrial cancer.

Menopausal status such as early menarche or late menopause is one of the risk factors for developing endometrial cancer. It is common that endometrial cancer occurs in postmenopausal women in Caucasians. However this is not the case for Chinese women. Our recent study reported that endometrial cancer also frequently (42\%) occurs in Chinese women before menopause [21].
Therefore in this study we also investigated the positivity of GPR30 in women with endometrial cancer taking into account menopausal status. In our current study we found that there was no difference in the positivity of GPR30 between subtypes of cancer in both premenopausal and postmenopausal women suggesting the positivity of GPR30 was not associated with menopausal status regardless of subtypes of cancer.

As an alternative estrogen receptor, studies have suggested that the expression of GPR30 is negatively correlated with ER expression [18, 22]. We have recently reported that the overall positivity of ER in endometrial cancer was $85 \%$ in Chinese population [6], which was similar with the overall positivity of GPR30 in our current study. We also reported a significantly lower positivity of ER in type 2 endometrial cancer in postmenopausal women, which may be associated with poorer prognosis [6]. However, in this study we found that the positivity of GPR30 was not associated with menopausal status regardless of subtypes of cancer. Other studies reported that GPR30 was expressed in up to $50 \%$ of breast cancer regardless of the positivity of ER, suggesting GPR30 and ER have an independent influence on estrogen responsiveness in breast cancer [23]. This prompted us to question whether in fact there is a negative correlation between GPR30 and ER positivity in endometrial cancer. In our current study we interestingly found that the positivity of ER was not different between cases with GPR30 positive and cases with GPR30 negative. In addition, our data also showed that the intensity of immunostaining of ER was not correlated with the intensity of immunostaining of GPR30. Therefore our data suggest that the immunostaining of GPR30 was not correlated with the immunostaining of ER in endometrial cancer, similar to breast cancer. 
There are however also important limitations of this study. Despite of the collection of samples over the study period in one women's hospital with total number of 128 cases, the number of cases, in particular the number of cases with type 2 endometrial cancer is small. To increase the power, the conclusions drawn from this study would need to be further studied with large sample size. In addition, the age of menopause was self-reported and data on disease progression and survival were not available in this study.

In conclusion, to our knowledge this is the first report comparing the positivity and immunostaining of GPR30 between type 1 and type 2 endometrial cancer taking into account menopausal status. We demonstrate that the overall positivity of GPR30 was $82 \%$ and there was no difference in the positivity and intensity of immunostaining of GPR30 between subtypes of endometrial cancer. We also found the positivity of GPR30 was not associated with menopausal status as well as with the positivity of ER in endometrial cancer. Type 2 endometrial cancer is commonly described as estrogen independent which suggests that estrogenic and antiestrogenic exposures would not be related to its risk. Obesity is associated with higher levels of circulating estrogens in postmenopausal women and with lower progesterone levels in premenopausal women. However, our current study further confirms that type 2 endometrial cancer is not completely estrogen independent, and suggests that type 1 and type 2 endometrial cancer may have similar pathogenesis. Therefore risk factors that are associated with estrogen such as obesity are also important for developing type 2 endometrial cancer. In addition, using hormones or hormone-blocking drugs to treat endometrial cancer which are used to treat endometrial cancer cell with hormone receptors may also apply to type 2 endometrial cancer.

\section{MATERIALS AND METHODS}

This study was approved by the Ethics Committee of Wuxi Maternity and Children Hospital, Nanjing Medical University of China. All patient-derived tissues were obtained with written informed consent. All methods were performed in accordance with the relevant guidelines and regulations.

\section{Study participants}

There were in total 128 women with a primary diagnosis of endometrial cancer who consented to donate the tissue for this study from January 2010 to December 2015 from Wuxi Maternity and Children Hospital, Nanjing Medical University of China. All data including age at diagnosis, self-reported age at menopause, parity and pathological findings of endometrial cancer were collected from the electronic based medical records of patients from the hospital.
The classification of type 1 and type 2 endometrial cancer was determined by pathological examination of biopsies, including cancer histologic subtypes and grades. We classified endometrioid and adenosquamous carcinoma with grade 1 and 2 as type 1 endometrial cancer. Clear-cell, serous, mucinous carcinoma and grade 3 endometrioid carcinoma were classified as type 2 endometrial cancers, according to the classification of the International Federation of Gynaecology and Obstetrics (FIGO).

Endometrial cancer was diagnosed first by a physical examination and then endometrial biopsy. The endometrial tissue was examined histologically for characteristics of cancer including types of cancer.

\section{Immunohistochemistry}

The immunostaining of ER and GPR30 in endometrial tissue $(n=128)$ was measured by immunohistochemistry on paraffin-embedded sections. Briefly, antigen retrieval was performed by treatment with citric acid ( $\mathrm{pH}$ 6.0) for 20 minutes. Non-specific antibody binding was blocked by incubating with $10 \%$ fetal calf serum for 20 minutes. Mouse anti-human ER (1:100, Dako, 1D5) or rabbit anti GPR30 polyclonal antibody (1:200, Abcam, ab154069) was added for 1 hour at room temperature. Sections were then washed with phosphatebuffered saline (PBS) and incubated with biotinylated anti-mouse/rabbit IgG (Dako, Denmark) for 30 minutes, and after washing sections were then incubated with streptavidin-conjugated horseradish peroxidase (Dako, Denmark) for 30 minutes. The antigen-antibody complexes were visualised using 3,3-Diaminobenzidine (DAB) and counterstained with haematoxylin. Negative controls were incubated with the irrelevant mouse/rabbit serum.

For ER positive, the cut-off point of $1 \%$ positive cells was considered as ER positive.

\section{Semi-quantitative analysis of immunohistochemical staining of GPR30}

15 images from each sample were taken with the microscope settings unaltered. Semi-quantitative analysis of GPR30 immunostaining in immunohistochemical images was undertaken using a previously published method based on the combination of staining intensity and the percentage of positive cells [24]. Briefly, no staining is scored as $0 ; 1-30 \%$ of positive cells scored as $1 ; 31-70 \%$ of positive cells as 2 ; and $71-100 \%$ of positive cells as 3. Staining intensity is rated on a scale of 0 to 3 , with 0 = negative; 1 = weak; $2=$ moderate, and $3=$ strong. The raw data were converted by multiplying the quantity and staining intensity scores. The final score points were presented as the average. Final score 0 , or 1 and 2 , or 3 and 4 , or 6 and 9 was considered as negative, $1+$, or $2+$ or $3+$ respectively. 


\section{Statistical analysis}

The statistical difference in positivity of ER and GPR30 in patients with type 1 or type 2 endometrial cancer in premenopausal and postmenopausal women and the correlation between ER and GPR30 positivity were assessed by the Fisher's exact test using the Prism software package (GraphPad Software Inc, San Diego, CA, USA) with $p<0.05$ being considered as statistically significant. Semi-quantitative analysis of immunohistochemical staining of GPR 30 between type 1 and type 2 was assessed $t$ test (non-parametric) using the Prism software package.

\section{Authors' contributions}

Wan JY: Sample collection and experiment performce; Yin YX: Sample collection and experiment performce; Zhao M: Clincial data collection, Shen F: Data analysis; Chen MX: Study design, Chen Q: Study design and manuscript writing.

\section{ACKNOWLEDGMENTS AND FUNDING}

This study was supported by a grant from The hospital of Obstetrics and Gynaecology, Fudan University. Authors thank all the patients who donated the tissues for this study.

\section{CONFLICTS OF INTEREST}

None of the authors have a conflicts of interest.

\section{REFERENCES}

1. Grady D, Gebretsadik T, Kerlikowske K, Ernster V, Petitti D. Hormone replacement therapy and endometrial cancer risk: a meta-analysis. Obstet Gynecol. 1995; 85:304-13.

2. Bokhman JV. Two pathogenetic types of endometrial carcinoma. Gynecol Oncol. 1983; 15:10-7.

3. Hoadley KA, Yau C, Wolf DM, Cherniack AD, Tamborero D, Ng S, Leiserson MD, Niu B, McLellan MD, Uzunangelov V, Zhang J, Kandoth C, Akbani R, et al, and Cancer Genome Atlas Research Network. Multiplatform analysis of 12 cancer types reveals molecular classification within and across tissues of origin. Cell. 2014; 158:929-44.

4. Srijaipracharoen S, Tangjitgamol S, Tanvanich S, Manusirivithaya S, Khunnarong J, Thavaramara T, Leelahakorn S, Pataradool K. Expression of ER, PR, and Her-2/neu in endometrial cancer: a clinicopathological study. Asian Pac J Cancer Prev. 2010; 11:215-20.

5. Kobel M, Atenafu EG, Rambau PF, Ferguson SE, Nelson GS, Ho TC, Panzarella T, McAlpine JN, Gilks CB, Clarke BA, Bernardini MQ. Progesterone receptor expression is associated with longer overall survival within high-grade histotypes of endometrial carcinoma:
A Canadian high risk endometrial cancer consortium (CHREC) study. Gynecol Oncol. 2016; 141:559-63.

6. Shen F, Gao Y, Ding J, Chen Q. Is the positivity of estrogen receptor or progesterone receptor different between type 1 and type 2 endometrial cancer? Oncotarget. 2017; 8:506-11. https://doi.org/10.18632/oncotarget.13471.

7. Wan J, Gao Y, Zeng K, Yin Y, Zhao M, Wei J, Chen Q. The levels of the sex hormones are not different between type 1 and type 2 endometrial cancer. Sci Rep. 2016; 6:39744.

8. Setiawan VW, Yang HP, Pike MC, McCann SE, Yu H, Xiang YB, Wolk A, Wentzensen N, Weiss NS, Webb PM, van den Brandt PA, van de Vijver K, Thompson PJ, et al, and Australian National Endometrial Cancer Study Group. Type I and II endometrial cancers: have they different risk factors? J Clin Oncol. 2013; 31:2607-18.

9. Thomas P, Pang Y, Filardo EJ, Dong J. Identity of an estrogen membrane receptor coupled to a $\mathrm{G}$ protein in human breast cancer cells. Endocrinology. 2005; 146:624-32.

10. Revankar CM, Cimino DF, Sklar LA, Arterburn JB, Prossnitz ER. A transmembrane intracellular estrogen receptor mediates rapid cell signaling. Science. 2005; 307:1625-30.

11. Szego CM, Davis JS. Adenosine 3',5'-monophosphate in rat uterus: acute elevation by estrogen. Proc Natl Acad Sci USA. $1967 ; 58: 1711-8$.

12. Prossnitz ER, Arterburn JB, Sklar LA. GPR30: A G proteincoupled receptor for estrogen. Mol Cell Endocrinol. 2007; 265-266:138-42.

13. Rae JM, Johnson MD. What does an orphan G-proteincoupled receptor have to do with estrogen? Breast Cancer Res. 2005; 7:243-4.

14. Ge X, Guo R, Qiao Y, Zhang Y, Lei J, Wang X, Li L, $\mathrm{Hu}$ D. The G protein-coupled receptor GPR30 mediates the nontranscriptional effect of estrogen on the activation of PI3K/Akt pathway in endometrial cancer cells. Int $\mathrm{J}$ Gynecol Cancer. 2013; 23:52-9.

15. He YY, Cai B, Yang YX, Liu XL, Wan XP. Estrogenic $\mathrm{G}$ protein-coupled receptor 30 signaling is involved in regulation of endometrial carcinoma by promoting proliferation, invasion potential, and interleukin-6 secretion via the MEK/ERK mitogen-activated protein kinase pathway. Cancer Sci. 2009; 100:1051-61.

16. Leblanc K, Sexton E, Parent S, Belanger G, Dery MC, Boucher V, Asselin E. Effects of 4-hydroxytamoxifen, raloxifene and ICI 182780 on survival of uterine cancer cell lines in the presence and absence of exogenous estrogens. Int J Oncol. 2007; 30:477-87.

17. Vivacqua A, Bonofiglio D, Albanito L, Madeo A, Rago V, Carpino A, Musti AM, Picard D, Ando S, Maggiolini M. 17beta-estradiol, genistein, and 4-hydroxytamoxifen induce the proliferation of thyroid cancer cells through the g proteincoupled receptor GPR30. Mol Pharmacol. 2006; 70:1414-23.

18. Smith HO, Leslie KK, Singh M, Qualls CR, Revankar CM, Joste NE, Prossnitz ER. GPR30: a novel indicator of poor 
survival for endometrial carcinoma. Am J Obstet Gynecol. 2007; 196:386. e1-9.

19. Prossnitz ER, Oprea TI, Sklar LA, Arterburn JB. The ins and outs of GPR30: a transmembrane estrogen receptor. J Steroid Biochem Mol Biol. 2008; 109:350-3.

20. Mahdi H, Schlick CJ, Kowk LL, Moslemi-Kebria M, Michener C. Endometrial cancer in Asian and American Indian/Alaskan Native women: tumor characteristics, treatment and outcome compared to non-Hispanic white women. Gynecol Oncol. 2014; 132:443-9.

21. Gao Y, Zhao M, Dai X, Tong M, Wei J, Chen Q. The prevalence of endometrial cancer in pre- and postmenopausal Chinese women. Menopause. 2016; 23:884-87.

22. Huang GS, Gunter MJ, Arend RC, Li M, Arias-Pulido H, Prossnitz ER, Goldberg GL, Smith HO. Co-expression of GPR30 and ERbeta and their association with disease progression in uterine carcinosarcoma. Am J Obstet Gynecol. 2010; 203:242 e1-5.

23. Filardo EJ, Graeber CT, Quinn JA, Resnick MB, Giri D, DeLellis RA, Steinhoff MM, Sabo E. Distribution of GPR30, a seven membrane-spanning estrogen receptor, in primary breast cancer and its association with clinicopathologic determinants of tumor progression. Clin Cancer Res. 2006; 12:6359-66.

24. Soslow RA, Dannenberg AJ, Rush D, Woerner BM, Khan $\mathrm{KN}$, Masferrer J, Koki AT. COX-2 is expressed in human pulmonary, colonic, and mammary tumors. Cancer. 2000; 89:2637-45. 